\title{
Adjuvant chemotherapy for patients with ypT0-2N0-category after neoadjuvant chemoradiotherapy for rectal cancer
}

\author{
JUE-FENG WAN ${ }^{1,2^{*}}$, LI-FENG YANG ${ }^{1,2^{*}}$, JI ZHU ${ }^{1,2}$, GUI-CHAO LI ${ }^{1,2}$ and ZHEN ZHANG ${ }^{1,2}$ \\ ${ }^{1}$ Department of Radiation Oncology, Fudan University Shanghai Cancer Center; ${ }^{2}$ Department of Oncology, \\ Shanghai Medical College, Fudan University, Shanghai 200032, P.R. China
}

Received November 17, 2015; Accepted August 4, 2017

DOI: $10.3892 / \mathrm{mco} .2017 .1426$

\begin{abstract}
Locally advanced rectal cancer patients with ypT0-2N0 have good prognosis and may not require as many cycles of adjuvant chemotherapy as patients with a poor (ypT3-4 or $\mathrm{N}+$ ) response. The aim of the present study was to evaluate the three-year disease-free and overall survival between patients with ypT0-2N0 rectal adenocarcinoma who received 0-3 cycles of 5-fluorouracil-based adjuvant chemotherapy and those who received $>3$ cycles. A total of 106 patients with locally advanced rectal cancer, classified as ypT0-2N0 after surgery at the Fudan University Shanghai Cancer Center (Shanghai, China) between 2006 and 2012, were identified. The patients were divided into two groups depending on the number of cycles of adjuvant chemotherapy: Group 1 received $0-3$ cycles $(n=32)$ and group 2 received $\geq 4$ cycles of adjuvant chemotherapy $(n=74)$. The three-year disease-free survival and overall survival rates were 86.8 and $93.1 \%$ for group 1 $(\mathrm{P}=0.633)$, and 88.5 and $96.8 \%$ for group $2(\mathrm{P}=0.381)$. No statistically significant difference was observed between the two groups, suggesting that patients with ypT0-2N0 status may not require more than three cycles of post-operative chemotherapy. Further evaluation in prospective studies is urgently recommended.
\end{abstract}

\section{Introduction}

Colorectal cancer is one of the most common cancer types in western countries and post-operative adjuvant chemotherapy (ACT) with 5-fluorouracil (5-FU)-based regimens is now the standard treatment modality for TNM stage III (and 'high-risk' stage II) colon cancer. By contrast, clinical practice guidelines for ACT of locally advanced rectal cancer are not based on

Correspondence to: Dr Zhen Zhang, Department of Radiation Oncology, Fudan University Shanghai Cancer Center, 270 Dong An Road, Shanghai 200032, P.R. China

E-mail: zhenzhang6@hotmail.com

${ }^{*}$ Contributed equally

Key words: rectal cancer, chemotherapy, good prognosis solid scientific evidence and the level of scientific evidence for sufficient benefit is much lower compared with colon cancer.

A meta-analysis of 21 randomized clinical trials (RCTs) by Petersen et al (1) revealed that ACT significantly reduced the risk of mortality and disease recurrence in patients with rectal cancer. However, among these 21 RCTs, only one used pre-operative chemoradiotherapy and almost all of these patients underwent curative resection of rectal cancer without pre-operative treatment. Things are more complicated as a result of the wide use of neoadjuvant chemoradiation for patients with locally advanced rectal cancer.

A secondary analysis of a clinical trial by the European Organisation for Research and Treatment of Cancer (EORTC) Radiation Oncology Group (EORTC 22921) showed that only patients with good prognosis (ypT0-2) benefited from ACT (2). Furthermore, a retrospective study by Fietkau et al (3) suggested that post-operative chemotherapy can be spared for patients whose tumors were downstaged to ypNO following pre-operative chemoradiation. These results suggested that the identification of pathological parameters after pre-operative chemoradiotherapy may serve as predictive markers to determine which patients may benefit from 5-FU-based chemotherapy.

The ADORE clinical trial revealed that co-treatment with oxaliplatin alongside 5-FU for adjuvant treatment of poor-response patients (ypT3-4 or N+) significantly improved their three-year disease-free survival (DFS) (4). Regarding patients with ypT0-2N0, evidence for the benefit of post-operative chemotherapy was limited.

As rectal cancer patients categorized as ypT0-2N0 have good prognosis, they may not require as many cycles of ACT as patients with poor response (ypT3-4 or N+). The aim of the present study was to compare the three-year DFS and overall survival (OS) between patients with rectal adenocarcinoma classified as ypT0-2N0 who received 0-3 cycles of 5-FU-based $\mathrm{ACT}$ and those who received $>3$ cycles.

\section{Patients and methods}

Patients. The Fudan University Shanghai Cancer Center Ethics Review Board (Shanghai, China) approved the protocol of the present study. Clinical records of the patients were anonymized and de-identified prior to analysis so that written informed consent was not required. A retrospective, consecu- 
tive cohort study of 112 locally advanced rectal cancer patients with ypT0-2N0M0 after surgery at the Fudan University Shanghai Cancer Center (Shanghai, China) between February, 2006 and December, 2012 was performed. Patients were identified from the institutional colorectal cancer patient database. Patients with concurrent inflammatory bowel disease, malignancy or distant metastasis, as well as those with hereditary colorectal cancer syndromes or prior history of malignancy or radiotherapy of the pelvis were excluded. A total of six patients were lost to follow-up and were therefore excluded to yield the final cohort of 106 patients.

Treatment. Pre-treatment clinical stage was assessed on the basis of magnetic resonance imaging or computed tomography (CT). Furthermore, pre-treatment biopsies were reviewed and diagnoses confirmed by gastrointestinal pathologists at the Shanghai Cancer Center (Shanghai, China). To exclude the presence of synchronous tumors, all patients were also subjected to full colonoscopic evaluation, and the distance of the tumor from the anal verge was determined by digital rectal examination and proctoscopy. Patients were subjected to chemoradiotherapy with a median radiotherapy dose of 50 Gy and concurrent administration of oxaliplatin and fluoropyrimidine. Surgery was generally performed 6-8 weeks following completion of chemoradiotherapy (for 4.5-6 weeks) and included low anterior resection or abdominoperineal resection using total mesorectal excision principles. Following surgery, all medically fit patients were given ACT consisting of FOLFOX, XELOX or Capecitabine over 4-5 months. Standard pathological tumor staging of the resected specimens was performed in accordance with the guidelines of the College of American Pathologists, with histopathological diagnosis performed by dedicated gastrointestinal cancer pathologists (5). The tumors were entirely embedded and serially sectioned for hematoxylin and eosin staining, and microscopic evaluation. Post-operative follow-up consisted of a routine physical examination alongside cross-sectional imaging and assessment of carcinoembryonic antigen every 3-6 months for the first 2 years after completion of treatment and every 6-12 months for 2 additional years thereafter. Whenever any symptom of disease occurred or elevated tumor marker levels were detected, CT scans of the pelvis, abdomen and chest, full colonoscopic evaluation, and/or positron emission tomography were immediately performed.

Statistical analysis. Sites of relapse were classified as local recurrence and distant failure. Local recurrence was defined as recurrence within the pelvis, including the tumor bed, perineal scar, anastomosis or regional lymph nodes. Distant failure was indicated as disease recurrence detected in the lung, liver, brain and other organs outside the pelvis. The Kaplan-Meier method was used to estimate the disease-free survival (DFS) and overall survival (OS) (6). Patients for whom treatment had failed were identified at the time of disease recurrence or mortality due to any cause to calculate the DFS. The OS duration was calculated from the beginning of chemoradiotherapy until the date of the last follow-up visit for patients still living or until the time-point the patient succumbed. The association between the estimated DFS or OS with each of the potential prognostic factors was assessed using the log-rank test (7). Multivariate analysis was performed using the Cox regression model (8). A two-sided statistical test and $\mathrm{P}<0.05$ was considered to indicate a statistically significant difference. PASW Statistics 13 (SPSS Inc., Chicago, IL USA) was used for statistical analysis.

\section{Results}

Patient population and tumor characteristics. A total of 106 patients who were treated for rectal cancer with by chemoradiation followed by surgery and optional ACT were included in the present study. The median patient age was 53 years (range, 28-76 years). The majority of patients were men $(67 \%)$. The median dose of radiation applied prior to survery was 50 Gy (range, 44-55 Gy), and $82 \%$ of the patients were concurrently treated with capecitabine and oxaliplatin, while the remaining $18 \%$ received investigational fluoropyrimidine-based combination regimens (e.g. with bevacizumab or irrinotecan). At a median of 7 weeks after completion of chemoradiotherapy, total mesorectal excision surgery was performed. Of the patients, $39(36.8 \%)$ were subjected to low anterior resection and 67 patients $(63.2 \%)$ received abdominoperineal resection. Depending on the number of cycles of ACT, the patients were divided into two groups: Group 1 $(n=32)$ received $0-3$ cycles, and group $2(n=74)$ received $\geq 4$ cycles. The patient characteristics are listed in Table I.

Recurrence and survival. The median follow-up duration was 36 months (range, 19-98 months) for the entire cohort. Of these, 11 patients $(10.4 \%)$ presented with a disease relapse $(5$ in group 1 and 6 in group 2), and 4 patients (3.8\%) succumbed to the disease ( 2 in group 1 and 2 in group 2). The three-year DFS rate was $86.8 \%$ for group 1 and $88.5 \%$ for group 2 $(\mathrm{P}=0.633)$, and the $\mathrm{OS}$ rate was $93.1 \%$ for group 1 and 96.8 for group 2 ( $\mathrm{P}=0.381$; Fig. 1).

Prognostic factors. The effects of age, sex, yp stage, the type of surgery, lymphovascular invasion, baseline stage, distance from anal verge and the cycles of ACT on DFS and OS were evaluated (Table II). Univariate and multivariate analyses revealed that the patient age was an independent prognostic factor for DFS ( $\mathrm{P}=0.040$ and $\mathrm{P}=0.044$, respectively).

\section{Discussion}

The once common problem of local recurrence in rectal cancer treatment has been overcome by total mesorectal excision surgery and radiotherapy. Distant metastasis, mainly to the liver and lungs, has now become the major cause of mortality following curative treatment of localized disease.

As in other solid tumor types, the aim of adjuvant systemic treatment of rectal cancer is to eradicate sub-clinical tumor micrometastases after the primary tumour has been surgically removed, with or without the use of radiotherapy. For stage III colon cancer, large randomized clinical trials have demonstrated a reduction in recurrence and improvement of OS with 5-FU plus oxaliplatin (9-13). However, while adjuvant therapy is universally recommended by guidelines for stage III colon cancer, there is currently no consensus regarding the optimal treatment for rectal cancer. 
Table I. Patient characteristics $(n=106)$.

\begin{tabular}{|c|c|c|c|c|c|}
\hline \multirow[b]{2}{*}{ Variables } & \multicolumn{2}{|c|}{ Group 1 (ACT 0-3) } & \multicolumn{3}{|c|}{ Group $2(\mathrm{ACT} \geq 4)$} \\
\hline & $\mathrm{n}$ & $\%$ & $\mathrm{n}$ & & $\%$ \\
\hline \multicolumn{6}{|l|}{ Age (years) } \\
\hline Median & \multicolumn{2}{|c|}{56} & \multicolumn{3}{|c|}{52} \\
\hline$<50$ & 8 & 25 & 31 & & 42 \\
\hline$\geq 50$ & 24 & 75 & 43 & & 58 \\
\hline \multicolumn{6}{|l|}{ Sex } \\
\hline Male & 19 & 60 & 52 & & 70 \\
\hline Female & 13 & 40 & 22 & & 60 \\
\hline \multicolumn{6}{|l|}{ Pathological type } \\
\hline Adenocarcinoma & 32 & 100 & 74 & & 100 \\
\hline \multicolumn{6}{|l|}{ Baseline stage } \\
\hline II & 10 & 31 & 12 & & 16 \\
\hline III & 22 & 69 & 62 & & 84 \\
\hline \multicolumn{6}{|l|}{ Distance from anal verge } \\
\hline$\leq 5 \mathrm{~cm}$ & 25 & 78 & 45 & & 61 \\
\hline$>5 \mathrm{~cm}$ & 7 & 22 & 29 & & 39 \\
\hline \multicolumn{6}{|l|}{ yp stage } \\
\hline TONO & 16 & 50 & 29 & & 40 \\
\hline T1-2N0 & 16 & 50 & 45 & & 60 \\
\hline \multicolumn{6}{|l|}{ Surgery } \\
\hline Low anterior resection & 8 & 25 & 29 & & 39 \\
\hline Abdominoperineal resection & 24 & 75 & 45 & & 61 \\
\hline Lymphovascular invasion & 1 & 3 & 3 & & 4 \\
\hline Perineural invasion & 0 & 0 & 1 & & 1 \\
\hline \multicolumn{6}{|l|}{ ACT } \\
\hline FOLFOX & 2 & 6 & 10 & & 14 \\
\hline XELOX & 17 & 53 & 52 & & 70 \\
\hline Capecitabine & 2 & 6 & 12 & & 16 \\
\hline None & 11 & 35 & 0 & & 0 \\
\hline Median ACT cycles (n) & \multicolumn{2}{|c|}{2} & \multicolumn{3}{|c|}{5} \\
\hline \multicolumn{6}{|l|}{ Follow-up duration (months) } \\
\hline Median & \multicolumn{2}{|c|}{39} & \multicolumn{3}{|c|}{37} \\
\hline Range & \multicolumn{2}{|c|}{$20-98$} & \multicolumn{3}{|c|}{$19-92$} \\
\hline
\end{tabular}

ACT, adjuvant chemotherapy.

A meta-analysis of 21 RCTs reported a significant reduction in the risk of mortality (17\%) and in the risk of disease recurrence $(25 \%)$ among patients with rectal cancer undergoing ACT as compared with those undergoing observation only (1). However, only one of these 21 RCTs contained patients treated with to pre-operative chemoradiotherapy and almost all of these patients underwent curative resection of rectal cancer without pre-operative treatment. However, with the wide use of neoadjuvant chemoradiation for patients with locally advanced rectal cancer, there is a complexity of factors determining the outcome.

Park et al (14) revealed that the treatment response to neoadjuvant chemoradiotherapy is an early surrogate marker and is correlated with the treatment outcome. Tumor response (complete vs. partial vs. poor) was associated with a five-year recurrence-free survival (90.5 vs. 78.7 vs. $58.5 \%$; $\mathrm{P}<0.001$ ) (14). Thus, patients with different degrees of tumor response after neoadjuvant chemoradiation may diversely benefit from the same ACT.

The EORTC 22921 trial did not confirm a significant DFS or OS benefit for adjuvant 5-FU-based chemotherapy for locally advanced rectal cancer (15). A second analysis of the EORTC 22921 trial was performed to assess whether any sub-set of patients who, after pre-operative radiotherapy or chemoradiotherapy and surgery, may benefit from adjuvant post-operative 5-FU/leucovorin chemotherapy. Exploratory analyses suggested that only patients with good prognosis (ypT0-2) benefit from ACT (2). Furthermore, a prelimi- 
Table II. Univariate and multivariate analyses of DFS and OS.

\begin{tabular}{|c|c|c|c|c|}
\hline \multirow[b]{2}{*}{ Variable } & \multicolumn{2}{|c|}{ Univariate analysis $(\mathrm{P})$} & \multicolumn{2}{|c|}{ Multivariate analysis $(\mathrm{P})$} \\
\hline & DFS & OS & DFS & OS \\
\hline Age ( $<50$ vs. $\geq 50$ years) & 0.040 & 0.104 & 0.044 & 0.138 \\
\hline Sex (male vs. female) & 0.726 & 0.458 & 0.573 & 0.273 \\
\hline $\mathrm{ACT}(\leq 3$ vs. $>4)$ & 0.633 & 0.381 & 0.424 & 0.230 \\
\hline yp stage (pCR vs. yp I) & 0.361 & 0.185 & 0.421 & 0.388 \\
\hline $\begin{array}{l}\text { Surgery (low anterior resection vs. } \\
\text { abdominoperineal resection) }\end{array}$ & 0.538 & 0.566 & 0.621 & 0.473 \\
\hline Lymphovascular involvement (+ vs. -) & 0.368 & 0.699 & 0.185 & 0.922 \\
\hline Distance from anal verge ( $\leq 5 \mathrm{vs} .>5 \mathrm{~cm})$ & 0.785 & 0.450 & 0.844 & 0.455 \\
\hline Baseline stage (II vs. III) & 0.724 & 0.890 & 0.957 & 0.625 \\
\hline
\end{tabular}

ACT, adjuvant chemotherapy; DFS, disease-free survival; OS, overall survival.
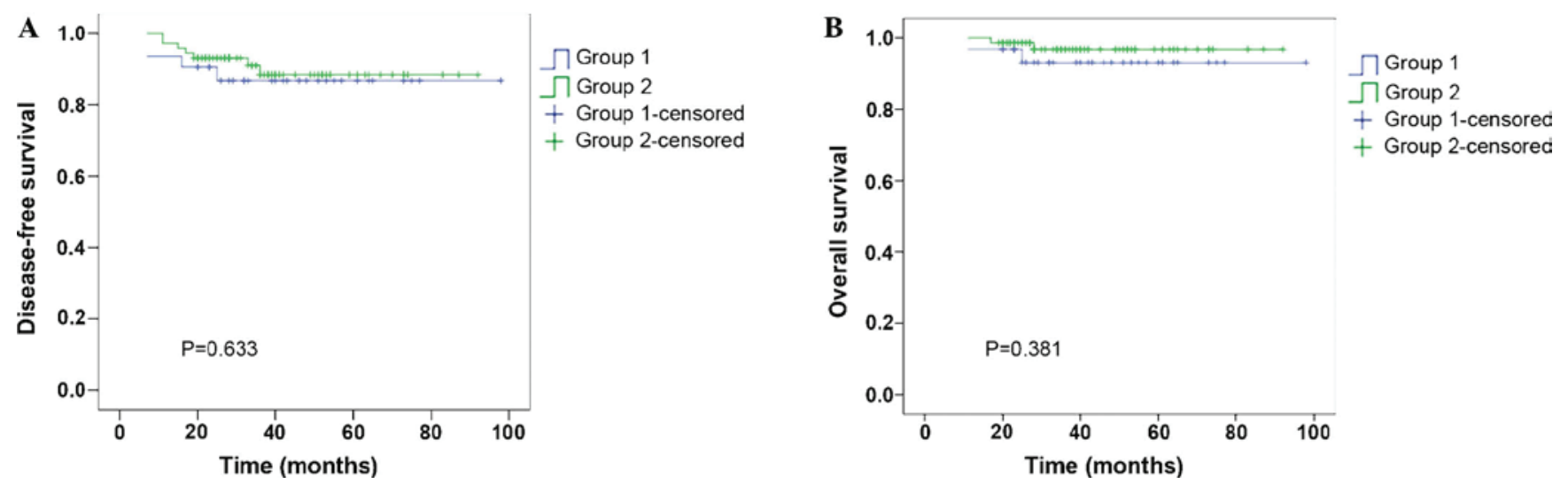

Figure 1. (A) Disease-free survival and (B) overall survival of the patients in group 1 (0-3 cycles of adjuvant chemotherapy) and group 2 ( $\geq 4$ cycles of adjuvant chemotherapy). The term censored indicates patients that were still alive at the time of last follo-up.

nary retrospective study by Fietkau et al (3) suggested that post-operative chemotherapy can be spared for patients whose tumors were downstaged to ypN0 after pre-operative chemoradiation.

These results suggested that the identification of pathological results after pre-operative chemoradiotherapy may serve as a predictive marker to determine which patients may benefit from 5-FU-based chemotherapy. A randomized phase II study (The ADORE) disclosed that the three-year DFS rate was $71.6 \%$ in the FOLFOX arm and $62.9 \%$ in the FL arm in patients with poor (ypT3-4 or N+) response with a hazard ratio (HR) of 0.657 (95\% confidence interval, 0.434-0.994; $\mathrm{P}=0.047)$ by intention-to-treat analysis (ASCO 2014) (4). The results of this study showed that the three-year DFS and OS of patients with good response (ypT0-2 N0) were not significantly different between those who received $0-3$ cycles of 5-FU-based ACT and those who received $>3$ cycles.

Four months of post-operative adjuvant chemotherapy are recommended, according to the treatment guidelines of the Fudan University Shanghai Cancer Center (Shanghai, China). However, the EORTC 22921 trial showed that chemotherapy was poorly tolerated after surgery for rectal cancer and $\sim 25 \%$ of patients did not receive any ACT (11). In addition, a phase II randomized study revealed that only $57 \%$ of patients received the full number of chemotherapy cycles after surgery (16). In the present study, $30 \%$ of patients were treated with 0-3 cycles of ACT due to patient refusal and toxicity.

In the present study, univariate and multivariate analysis revealed that age was an independent prognostic factors for DFS ( $\mathrm{P}=0.04$ and $\mathrm{P}=0.044$, respectively) and elderly patients had a better three-year DFS. Various studies have reported poorer prognosis among young patients with colorectal cancer when compared with elderly patients because of more advanced disease and poor tumor differentiation at diagnosis (17-23). By contrast, a recent retrospective study showed that young patients with non-metastatic colorectal cancer after surgery had better long-term survival (21). This may in part be due to their good performance status and suitability for chemotherapy (24-26).

The present study was a retrospective study and had therefore certain limitations. For example, the number of patients required for adequate statistical power was not 
determined and no final conclusion could be drawn as it was a retrospective study. In addition, as the evaluation of the potential benefit of total neoadjuvant treatment in the management of locally advanced rectal cancer remains to be fully determined, it remains elusive whether pre-operative total chemoradiotherapy is superior to the present method with regard to tolerance and treatment outcome (27). Future studies, ideally as collaborative efforts among cancer centers, should aim to resolve these questions.

Regarding patients with ypT0-2N0 status after neoadjuvant chemoradiation, the results of the present study reported that no statistical difference was present between patients treated with 0-3 cycles of ACT and those treated with $>3$ cycles. These results indicated that these patients may not require $>3$ cycles of ACT. Further evaluation in prospective studies is urgently recommended.

\section{References}

1. Petersen SH, Harling H, Kirkeby LT, Wille-Jørgensen P and Mocellin S: Postoperative adjuvant chemotherapy in rectal cancer operated for cure. Cochrane Database Syst Rev 3: CD004078, 2012.

2. Collette L, Bosset JF, den Dulk M, Nguyen F, Mineur L, Maingon P, Radosevic-Jelic L, Piérart M and Calais G: Patients with curative resection of c T3-4 rectal cancer after preoperative radiotherapy or radiochemotherapy: Does anybody benefit from adjuvant fluorouracil-based chemotherapy? A trial of the European organisation for research and treatment of cancer radiation oncology group. J Clin Oncol 25: 4379-4386, 2007.

3. Fietkau R, Barten M, Klautke G, Klar E, Ludwig K, Thomas H, Brinckmann W, Friedrich A, Prall F, Hartung G, et al: Postoperative chemotherapy may not be necessary for patients with ypN0-category after neoadjuvant chemoradiotherapy of rectal cancer. Dis Colon Rectum 49: 1284-1292, 2006.

4. Hong YS, Nam BH, Kim KP, et al: Adjuvant chemotherapy with oxaliplatin/5-fluorouracil/leucovorin (FOLFOX) versus 5-fluorouracil/leucovorin (FL) for rectal cancer patients whose postoperative yp stage 2 or 3 after preoperative chemoradiotherapy: Updated results of 3-year disease-free survival from a randomized phase II study (The ADORE). J Clin Oncol 32 (Suppl; Abstract no. 3502): 5S, 2014

5. Washington MK, Berlin J, Branton P, Burgart LJ, Carter DK, Fitzgibbons PL, Halling K, Frankel W, Jessup J, Kakar S, et al: Protocol for the examination of specimens from patients with primary carcinoma of the colon and rectum. Arch Pathol Lab Med 133: 1539-1551, 2009

6. Kaplan EMP: Nonparametric estimation from incomplete observations. J Am Stat Assoc 53: 457-481, 1958.

7. Mantel N: Evaluation of survival data and two new rank order statistics arising in its consideration. Cancer Chemother Rep 50 163-170, 1966

8. Gill RD: Multistate life-tables and regression models. Math Popul Stud 3: 259-276, 1992.

9. André T, Boni C, Mounedji-Boudiaf L, Navarro M, Tabernero J, Hickish T, Topham C, Zaninelli M, Clingan P, Bridgewater J, et al: Oxaliplatin, fluorouracil and leucovorin as adjuvant treatment for colon cancer. N Engl J Med 350: 2343-2351, 2004.

10. Wolmark N, Wieand S, Kuebler JP, et al: A phase III trial comparing FULV to FULV+oxaliplatin in stage II or III carcinoma of the colon: Results of NSABP Protocol C-07. J Clin Oncol 23 (June 1 Suppl): LBA3500, 2005.
11. Kuebler JP, Wieand HS, O'Connell MJ, Smith RE, Colangelo LH, Yothers G, Petrelli NJ, Findlay MP, Seay TE, Atkins JN, et al: Oxaliplatin combined with weekly bolus fluorouracil and leucovorin as surgical adjuvant chemotherapyfor stage II and III colon cancer: Results from NSABP C-07. J Clin Oncol 25: 2198-2204, 2007.

12. André T, Boni C, Navarro M, Tabernero J, Hickish T, Topham C, Bonetti A, Clingan P, Bridgewater J, Rivera F and de Gramont A: Improved overall survival with oxaliplatin, fluorouracil and leucovorin as adjuvant treatment in stage II or III colon cancer in the MOSAIC trial. J Clin Oncol 27: 3109-3116, 2009.

13. Haller DG, Tabernero J, Maroun J, de Braud F, Price T, Van Cutsem E, Hill M, Gilberg F, Rittweger K and Schmoll HJ: Capecitabine plus oxaliplatin compared with fluorouracil and folinic acid as adjuvant therapy for stage III colon cancer. J Clin Oncol 29: 1465-1471, 2011.

14. Park IJ, You YN, Agarwal A, Skibber JM, Rodriguez-Bigas MA, Eng C, Feig BW, Das P, Krishnan S, Crane CH, et al: Neoadjuvant treatment response as an early response indicator for patients with rectal cancer. J Clin Oncol 30: 1770-1776, 2012.

15. Bosset JF, Collette L, Calais G, Mineur L, Maingon P, Radosevic-Jelic L, Daban A, Bardet E, Beny A and Ollier JC: Chemotherapy with preoperative radiotherapy in rectal cancer. N Engl J Med 355: 1114-1123, 2006.

16. Fernández-Martos C, Pericay C, Aparicio J, et al: Phase II, randomized study of concomitant chemoradiotherapy followed by surgery and adjuvant capecitabine plus oxaliplatin (CAPOX) compared with induction CAPOX followed by concomitant chemoradiotherapy and surgery in magnetic resonance imaging-defined, locally advanced rectal cancer: Grupo cancer de recto 3 study. J Clin Oncol 28: 859-865, 2010.

17. Taylor MC, Pounder D, Ali-Ridha NH, Bodurtha A and MacMullin EC: Prognostic factors in colorectal carcinoma of young adults. Can J Surg 31: 150-153, 1988.

18. Marble K, Banerjee S and Greenwald L. Colorectal carcinoma in young patients. J SurgOncol 51: 179-182, 1992.

19. Cusack JC, Giacco GG, Cleary K, Davidson BS, Izzo F, Skibber J, Yen J and Curley SA: Survival factors in 186 patients younger than 40 years old with colorectal adenocarcinoma. J Am CollSurg 183: 105-112, 1996.

20. Li Q, Cai G, Li D, Wang Y, Zhuo C and Cai S: Better long-term survival in young patients with non-metastatic colorectal cancer after surgery, an analysis of 69,835 patients in SEER database. PLoS One 9: e93756, 2014.

21. O'Connell JB, Maggard MA, Livingston EH and Yo CK: Colorectal cancer in the young. Am J Surg 187: 343-348, 2004

22. Ben-Ishay O, Brauner E, Peled Z, Othman A, Person B and Kluger Y: Diagnosis of colon cancer differs in younger versus older patients despite similar complaints. Isr Med Assoc J 15: 284-287, 2013.

23. Karsten B, Kim J, King J and Kumar RR: Characteristics of colorectal cancer in young patients at an urban county hospital. Am Surg 74: 973-976, 2008.

24. Goodwin RA and Asmis TR: Overview of systemic therapy for colorectal cancer. Clin Colon Rectal Surg 22: 251-256, 2009.

25. Chew MH, Koh PK, Ng KH and Eu KW: Improved survival in an Asian cohort of young colorectal cancer patients: An analysis of 523 patients from a single institution. Int J Colorectal Dis 24: 1075-1083, 2009

26. Serra-Rexach JA, Jimenez AB, Garcia-Alhambra MA, Pla R, Vidán M, Rodríguez P, Ortiz J, García-Alfonso P and Martín M: Differences in the therapeutic approach to colorectal cancer in young and elderly patients. Oncologist 17: 1277-1285, 2012.

27. Nilsson PJ, van Etten B, Hospers GA, et al: Short-course radiotherapy followed by neo-adjuvant chemotherapy in locally advanced rectal cancer-the rapido trial. BMC Cancer 13: 279, 2013. 